\title{
Profile Interview with Dr. Luckson Dullie
}

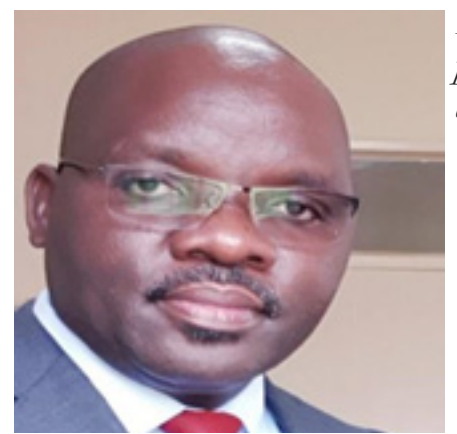

Haules Zaniku (HZ), MMJ intern, speaks to Dr. Luckson Dullie (LD) on his professional background, position as the Executive Director for Partners In Health (PIH) and on the role of PIH in addressing HIV and Non Communicable Diseases burden in Malawi.

HZ: Please, tell me about yourself and your professional background?

LD: I am a Malawian trained doctor who graduated from the College of Medicine (University of Malawi) in 2001. I did my internship at Queen Elizabeth Central Hospital (Q.E.C.H) from 2001 to 2003. My career interest became biased towards internal medicine and obstetrics after I had finished my internship. My other interest was in surgery but wasn't deep like the two I had mentioned earlier.

Taking into account that studying at the College of Medicine was intensive, I had fears to continue my studies soon after the internship. I decided to take a break so that I could specialise later. The break took me to Malamulo Adventist Hospital where I worked from 2003. My hope was to work for a short time and return to college for specialization.

My experience at Malamulo made me realize that hospitals in rural areas have few Doctors, so medical doctors assume some role of a consultant and become mentors to clinical officers. At that time the hospital had three Doctors. I later started teaching at the Malamulo College of Health Sciences as well.

Apart from my general services, I was also working in Out Patients Department, internal medicine, obstetrics, gynecology and surgery departments which I thought was too much. Regardless of this pressure I appreciated working in a rural setting because I got experience from various departments.

Between 2004 and 2005, I thought of moving on with life and career although I didn't have a direction as to where I wanted to move to. I wanted to explore into an area that would help me specialize in a variety of areas so that I could fit in multiple departments. I eventually went to Lesotho, Maluti Adventist Hospital, which is a sister hospital to Malamulo Adventist Hospital. My intention was to use their environment to develop my surgical skills with the senior medical personnel working there. Initially the hospital was doing more surgeries than Malamulo. They were also handling more HIV and AIDS cases so I knew I would still practice in public health.

Whilst in Lesotho, my fellow students and I started a conversation of what to call this inclusive program. We suggested several different names like general medicine for rural practice up until when we agreed to call it Family Medicine. We further explored this with the staff at the
University of Free State that had this department and truly the name fitted very well.

In March 2006, I registered in the Family Medicine program which I finished in 2009. This is how I ended up in Family Medicine. After coming back, I continued working with the Adventists before joining the College of Medicine in 2011. At that time the College had just started the Department of Family Medicine as a product of the Internal Medicine Department. Within three years the department developed an undergraduate curriculum of the MBBS (Bachelor of Medicine and Bachelor of Surgery) programme and also got an approval to have the Master of Medicine (MMED) in Family Medicine.

\section{HZ: What motivated you to do Family Medicine?}

LD: Family Medicine has evolved over time. First as I said, I was looking at myself in a setting where I needed to have multiple skills. I realized that I needed public health, internal medicine, surgery and obstetrics and gynecology. I needed to master all because of the setting in which I was. That's basically the clinical component of Family Medicine. You need to have appropriate skills, be trained to be able to function in those areas. Overtime, as we worked on the student rotation and also the development of the M Med curriculum in Family Medicine, the concept of the multiskilled clinicians became more important. What became more important also were things like leadership for primary health care. Looking at the setting we're in, the district health system is a platform in which our primary health care is delivered. But when we focus on the leadership at a district level, usually there are Doctors that have recently graduated from College of Medicine who are not adequately prepared to manage this kind of a system yet. They develop and grow into it yes, but they are not initially prepared for that particular function. This aspect of Family Medicine started to become more and more important. The curriculum that we eventually developed was not just for clinical elements of the functional Family Medicine but also took into consideration the district health system and provides the clinical leadership.

HZ: What do you think has been the role of Family Medicine as a program so far in Malawi?

LD: In Malawi, Family Medicine has really changed medical training in general with the sense that this is one of the rotations that takes students out of their conventional training platforms which has been in the central hospitals. The whole idea of posting fresh graduates to district hospitals and mission hospitals is that they should be able to see and experience a different range of pathologies. When we are in a tertiary hospital or a central hospital for example, there is only one kind of a spectrum of conditions that you can expect. All the cases are usually referred and 
are more serious. This in turn skews the understanding of medicine because then you think that patients who come in walking are not sick and they don't need to see a Doctor. Then this brings in a thinking that the lowest level of health care workers in the system is where these patients should be seen. So the ambulatory patients are seen by the lowest trained group such as the medical assistants but with Family Medicine we want to change that view, that even when the students are being trained they can experience different range of pathologies so that their approach to patients can be different.

In addition, these Doctors are trained either in Lilongwe or in Blantyre, but the majority of the population in Malawi is in rural areas and yet we expect these people to go and work in the rural when they have no experience. There are many studies out there that have shown that where you are trained will influence your future career projections and so this program was deliberately designed to provide the opportunity for students to experience medicine in the rural setting. For instance, students who come and spend four weeks with us here in Neno would usually ask for opportunities to come back and do different sets of time. I think what triggers this is the contribution from Family Medicine where students are exposed to a rural area setting. There is also life in rural areas and this is where you have all sorts of interaction with other different professionals, see the possibilities of others and grow professionally.

From a health system perspective, we would be able to see the contribution of Family Medicine in the management of the district health system as a whole and not just from a leadership management perspective but also even in terms of clinical outcomes. The quality of service provided at a district level, the quality of referrals being made and the whole kind of governance and structures as well. So those are benefits that we would anticipate as Family Medicine continues to grow.

HZ: As one of the pioneers for Family Medicine, what were your contributions?

LD: So by the time I was coming back in 2009, they had already been conversations about a Family Medicine rotation. In fact the MBBS curriculum had just been revised before I returned from Lesotho and a 6 weeks Family Medicine rotation was included in the fourth year of MBBS program. The College was actively looking for someone to lead that program and I came in the midst of all that. On my arrival, I started working with groups that were facilitating this process at the College of Medicine and we had conversations with a close colleague of mine, a family physician too from South Africa. At that time, Family Medicine was a unit in internal medicine and from 2011 I was program coordinator for Family Medicine. The time we transitioned from a unit to a department was the same time the college was transitioning from a single faculty college to the now the multi-faculty institution.

During the transition, there was an agreement that Family Medicine program should join the School of Public Health mainly because Family Medicine is not purely internal medicine, nor surgical, nor gynae but a combination of all that. Apart from the combination of those clinical components, we also have a lot of community oriented aspects. So we see ourselves as sitting between the clinical departments and public health. The time I was leaving the college, the department had already been established and I sat in as an interim head for a bit of time before I joined Partners In Health.

HZ: You are actively involved in understanding Primary Health Care (PHC) in Malawi, what have been the achievements, challenges met and what is the future direction as a country?

LD: I think we have made some progress. If you go back decades, one thing we have tried as a country is to improve health coverage i.e. geographical access to facilities has improved overtime. The idea of an essential health package though it has got challenges, I think it has helped us to focus on some package.

Over the last five years there has been a discussion around health reforms such as central hospitals becoming autonomous and other necessary reforms. There has also been discussions about reforms at a district level but we haven't heard or seen anything with primary health care and yet this is the first entry level for a patient into the health system.

If you stop for a moment to count how many patients visit a health centre around the country, our experience here in Neno is that we have 120 patients per facility in a normal day. So for Neno which has 14 health centres this will mean that more people will be seen in a day. And as I said that we continue to maintain the notion that because these patients are ambulatory then it is fine that the patients should firstly be seen by the medical assistants. This is a challenge. The medical assistants could be the lowest level of professionals that are primary care providers in the Southern Africa Development Community (SADC) or probably all across. Most countries have changed to higher level of clinical officer.

Another challenge is that health centers are the least supported in the health system and most of the times the health center is the last to be considered. There is more that we can do, for example, if you go in literature, there is a lot of evidence that a functional health system needs a strong primary health care platform to be effective. We are missing out by not rethinking our primary health system in the way that we can redesign it and the way that we deliver it. Out of interest, we don't have a primary health care policy. What we have are elements of it. If we go for example in the health sector strategic plan you will see some language of primary health care in it but most of is just to make somebody happy.

We say we are signatories of primary health care declarations and we need to show that we are following up on those kinds of commitments but in reality, I don't think there has been much progress. We have to debate on how we want primary health care to be delivered and there must be a clear policy on what we want to do, how we are going to deliver it, who is going to deliver it and how we are going to fund it. Central hospital receive funding directly and we expect the money that comes through local councils to fund primary health care. The reality is that the biggest chunk of that money ends at the district hospital. My suggestion is that the whole primary health care needs to be a separate level so that it should receive separate attention that it requires.

HZ: Are there any discussions to have this policy for primary health care?

LD: We've made discussions with bodies like the Society of Medical Doctors and the most recent with the College of Physicians and Surgeons in Malawi. The discussions are that 
once the primary health policy is established we should have different professions forming their own colleges under this umbrella body so that each profession or specialty can define its own space and have an oversight function.

Processes are now underway to organize ourselves as an Association of Family Physicians. Once this professional association is established it will become a voice that can contribute in policy development, implementation and oversight for this profession. To me this is an exciting piece of progress that we see coming through and we need to move quite fast so that we can provide appropriate guidance in as far as primary health care is concerned.

\section{HZ: Tell me a brief history of Partners in Health (PIH) in Malawi?}

LD: PIH has been in Malawi since 2007. Initially there was a kind of consortium of organizations and it included Clinton -Hunter foundation and Partners In Health (PIH). ClintonHunter Foundation's focus was much on health systems strengthening while PIH is an implementing NGO.

When Neno was separated from Mwanza in 2002, its health system had a network of rural health centers but it had no district hospital. It only had health staff typical to that of health centers. Being a very disadvantaged rural population that needed help to build up the health system, the Clinton -Hunter provided the initial funding while PIH did the implementation. Two years down the line, the ClintonHunter pulled out of the consortium. So starting from about 2009 it's been purely PIH and we have continued to receive support from our main office in Boston, USA where the organisation is based.

There has been more infrastructure development like building of the district hospital, community hospital and some health centres supported with human resources from doctors, nurses, clinical officers and laboratory personnel. As $\mathrm{PIH}$, an important model that we use is that we don't own or run a facility in any of the sites we are working. All we do is to support the public sector in its functions to deliver the services. So across the district we support the services that the public sector is supposed to deliver.

The time we were coming to Neno, we priotised HIV since it was out of necessity and it was a major problem that needed to be tackled. So we focused on HIV at that time. Our delivery model included the medical side such as providing medicines as well as social support. We have a department called social and economic rights which provides some of the social support that patients need.

Overtime we have integrated Non-Communicable Diseases (NCDs) and HIV. Experience has shown that we usually have good outcomes in many ways for instance with the HIV cohort but we had not done that well with the NCD cohort. This made us to integrate an ART clinic and the NCD clinic and then have what we call an integrated chronic care clinic which brings together services for HIV patients, hypertension, diabetes and asthma. It is now three years since we started this integrated chronic care clinic and all these conditions are seen through the same platform.

\section{HZ: How did you feel when you were appointed as the} Executive Director for PIH, Malawi?

LD: It was quite an interesting transition. I come from a background of being purely clinical and with two or three years in the academia at College of Medicine before I became the Executive Director for PIH. It's a very big organisation.
We have a staff complement of about 320 employees and a budget of around 4 to 4.5 million USD per year. It was a challenging transition but it was all easier because of my previous experience not just as a clinician but also with the concept of Family Medicine. In Family Medicine, we use patient-centered model of delivery whereby we think of every person who comes as a whole person and not looking at the disease in the person but looking at the person with the disease.

It's from these concepts that align the philosophy that drives PIH. So with PIH, we look at the preferential option of the poor and we focus on the poor and the vulnerable people. But interestingly, in the delivery platform for PIH we have the medical, social support and community health components and we see how these transpose so easily form a holistic people centered approach philosophy that is Family Medicine. So it became so easy for me to fit into the system on one hand. On the other hand, I saw it as an opportunity because while I was at COM I was always thinking of the future to say in 5 years when we graduate the first cohort of Family Physicians this is what they should be like.

This opportunity to work with PIH fast tracked my thinking because it gave me the opportunity to be that person so I stopped thinking in 5 years' time, but I fast tracked that thinking to say now I am that person working in the district and providing that leadership and so it became quite exciting to think about it that way. And it was basically a combination of all those thinking processes along Family Medicine lines and to work with an organisation which out of philosophical point of view operates with the same kind of principles.

HZ: What comprises your responsibilities as the Director?

LD: If we look at what the PIH tries to achieve, like I said, essentially is to support the public sector to deliver on its mandate. But we have different faces on that, because on one hand we deliver the services directly or support the public sector to deliver those services but we also use the experiences that we learn through the delivery of those services to advocate for change. We also use the evidence that we generate like in Neno to be thought of as partners in the policy development to change some things that would impact on a wider scale. So there is service delivery, there is advocacy, there is research and there is also capacity building. We provide trainings whether within PIH or the Ministry of Health $(\mathrm{MoH})$ side and we have different kinds of students who also come here. Those are the main areas that we focus on and my role is to lead the organisation in its strategic thinking and delivery in those platforms. We need to deliver and I am responsible to make sure that we continue to deliver on that mandate. I also have to ensure that as an organisation we continue to exist and that's in terms of the oversight whether in terms of the financial management and human resources development.

HZ: What important work is PIH currently undertaking in the HIV and NCDs care in Malawi, and how is it contributing towards addressing the HIV and NCDs burden in the country?

LD: We implement programs not just to bring quality where it is needed but also to use it is as a platform of learning. So we have used a lot of lessons for example, in the HIV space we have been part and parcel of the technical working group at $\mathrm{MoH}$ in HIV and we have been contributing to the 
whole thinking of how HIV care continues to be delivered efficiently and effectively in Malawi. When we look at HIV outcomes, Neno will always be higher up whether it's in terms of retention in care, survival on treatment, minimizing loss to follow up and other parameters.

As I said, the integration of the NCDs into HIV has also been kind of a major shift. You hear a lot about integration in different ways but I think what we have is a true manifestation of how integration should really happens. We have all these patients being seen physically in the same space by the same people and provided the same level of quality care.

Decentralization of the system has also been another thing. This is one of the places in Malawi where we have the integrated chronic care clinic at every health facility in the district. So we have NCD and HIV patients screened pretty much across the district. To have a way in which we deliver this platform of care across the district is something that we have contributed. We work very closely with the $\mathrm{MoH}$ NCD unit and in fact PIH has been a co-chair of NCDs and injuries commission in Malawi.

There is a report which was written up on common NCDs and Injuries amongst the poorest and this has helped to shape the national action plan and implementation plan of the NCD unit. For the past 3 years, we have a technical assistant attached to the NCD unit which allows us to contribute to policy discussions as well as continue to be an important implementation organisation at the very lowest level.

This has enabled us to learn directly from implementation to the policy discussions. It's been a very fruitful journey. You will recall at the United Nations Assembly where Malawi received an award for progress in NCDs. We quietly applauded ourselves that we contributed in a significant way towards this achievement without standing on the hilltop about it but we know the work that we have contributed at the MoH NCDs unit.

HZ: Are there plans to scale-up the services to other district in Malawi?

LD: It's a question I receive all the time. Sometimes I struggle with it. Sometimes I wake up and I want us to be in Blantyre, Nsanje, Chikwawa and across different settings. But again I have to remember why we are here and one of the most important things that help to bring me back to where we should be is that even though we are an implementing organisation but one very important element of that is to advocate for change.

There is need to balance how much of what we do to be significant enough to meaningfully contribute to policy discussions so that they become our main focus. We use Neno to develop what we have come to call a model for district health services. And then to use that model for scaling up. The scaling up does not necessarily mean that PIH should then go to Phalombe and start working there but that we can contribute to those policy discussions so that certain elements of what we do become policy. That's the scaling up that we are looking at so that what we do that contributes to success should be incorporated in the way of doing things in Malawi and beyond.

HZ: As someone who has worked with PIH for quite some time now, are there challenges you have met in your work and how have you managed to overcome them?

LD: The biggest challenge we face is a positive tension between being ambitious in what we need to deliver and balancing that against the resources we need for the delivery of that particular package. In my experience we struggle with requests such as an ambulance that doesn't have tires, fuel or the generator that doesn't have fuel, the patients that don't have food or the hospital cannot be cleaned because there are no cleaning supplies.

And so as an organisation that literally seats alongside the Ministry of Health we clearly see those challenges. The balance of how we can manage those kinds of needs against the service delivery or innovations that we were talking about as well as balancing the needs and the funds from public sector to the innovations that we want to achieve, the quality that we want to deliver is a big challenge. There is a big gap. It has been a constant struggle, for example whether we are going to put in money for pathology to send the biopsies for diagnostics or are we going to buy clinic supplies? Whether we want to have two clinical officers instead of having one medical assistant at a health center?

One of the ways we have been able to survive is that PIH is not a project oriented organisation. We don't come and say we will only work for two years then we fold up and we go. As a principle, PIH forms a long term commitment with the partners we work with, so we have a long term commitment with the Ministry of Health. We have been here for 13 years. We have learned a lot about how to juggle in those never ending priorities. We are able to learn about the cycle of poverty that the public sector goes through during the fiscal year and we can manage resources at different points within the year. Our long term commitment is a strong factor in bringing about the success that we see and being able to maintain and sustain the relationship we have with the public sector

\section{HZ: What can you say are the factors behind the success of PIH, Malawi?}

LD: Building up on the long term commitment is a strong factor. We have built relationships with people and with leaders in the ministry at all levels beginning from Capital Hill, all the way to the local partners right here.

Another reason for success is probably because of our source of funding within PIH. The bigger chunk of money in PIH comes from individual donors and these are people that will give different amounts of money. This means that our money doesn't come as already committed like of a typical NGO setting where they would say this is HIV money therefore we can't use it for anything else. The relationship we have with our donors is that they give the money with confidence and trust that we will make best of it. As a leader here, if I see that buying food for the patients is an important thing at that point in time then I will do that. If buying tires is what we need at that point, then we can make those kinds of decisions. So to have the flexibility in the use of funds that is not limited to projects restricted allows us to be flexible in a way that we can respond to challenges as they come.

\section{HZ: What outstanding life experiences or achievements are you proudest of?}

LD: From a personal perspective as I reflect from the past several years, making the decision to live in Neno together with my whole family will always stand out as life changing moment to me. Every time I meet my friends they will go like 'are you still in Neno?' I am in my $6^{\text {th }}$ year in Neno and having been able to do that to me has been so great. I 
thought I understood poverty or how a bad road looks like until I came here. Staying here has changed my perspective in a lot of ways. One thing I have been able to do while in Neno is that I have been able to finish my PhD. I just came back from a successful defense on the $6^{\text {th }}$ of February 2020. To be able to do that while in Neno and while maintaining my full time job and full time commitment also as a husband has been a unique experience. I thank God that we are done with that and now we can focus on other things.

HZ: What are your hobbies and things you are passionate about?

LD: Firstly, I like to be close to my family. During weekends or on public holidays I just stay home. Spending time with my family is what I love doing. Secondly, the environment here is so beautiful so I do morning walks, on average 6-7 kilometers per day. The air is so fresh and this time of the year is beautiful and green. It gives you time to think and plan for the day. And thirdly, I love to sing, I have been a choir member everywhere. But I have always been a member of Malamulo Hospital choir. Sometimes I make trips to Malamulo just for practice. I continue as much as possible to sing in this group.
HZ: Lastly, what is your vision for PIH, Malawi?

LD: What I see us doing together with the Ministry is to develop Neno as a true model of district health service delivery and to be able to show that through outcomes, be it clinical outcomes, patient experiences and all that. I would like to see us becoming a true partner in policy development in as far as district health services are concerned. So I dream of having a centre for primary health care research and implementation here in Neno.

\section{HZ: Do you have some last words?}

LD: Thank you for this opportunity to have this conversation and also to express the aspiration that we can make meaningful contributions to the health system in a way that changes us as a country but also manifests itself into meaningful opportunities, developments and improvements at the individual level as well. Thank you so much for the opportunity. 